\title{
\&UIIBRAGEC

\section{ANÁLISE DAS LIVES ENQUADRADAS NA ÁREA DE GESTÃo E ECONOMIA DA CONSTRUÇÃO REALIZADAS DURANTE A PANDEMIA DA COVID-19 ${ }^{1}$}

\section{CRUZ, Herbert Melo (1); AMARAL, Tatiana Gondim do (2); SANTOS, Davi Nascimento dos (3); ALVES, Mariana Ribeiro Saboia (4); SANTOS, Débora de Gois (5); HEINECK, Luiz Fernando Mahlmann}

(1) Instituto Federal de Sergipe, hmc_014@ hotmail.com, (2) Universidade Federal de Goiás, tatianagondim@ufg.br (3) Instituto Federal de Sergipe, davi.santos98@academico.ifs.edu.br (4) Universidade Federal de Goiás, marinasaboia@ discente.ufg.br (5) Universidade Federal de Sergipe, deboragois@yahoo.com.br (6) Universidade Estadual do Ceará, luiz.heineck@uece.br

\begin{abstract}
RESUMO
A pandemia da Covid-19 modificou repentinamente as relações entre pessoas, disseminação de conhecimento e consequentemente relações de ensino-aprendizagem. Diante disso, lives/webinários foram realizadas durante a pandemia, através das redes sociais e protagonizada por diversos atores, é válido questionar a relevância dessas lives para a popularização do conhecimento da Gestão e Economia da Construção (GEC). O objetivo deste trabalho foi catalogar as lives enquadradas nesta área e realizar uma análise qualitativa das mais visualizadas. Logo, foi realizado um levantamento das lives no Youtube, categorizando-as nas linhas temáticas da GEC e estabelecendo um ranking das mais visualizadas. Estas principais lives passaram por um processo avaliativo por especialistas da área, utilizando uma Ficha de Avaliação. Como principais resultados e contribuições, foram catalogadas 144 lives, enquadradas nas 14 linhas temáticas da GEC, foram obtidas 50 avaliações de especialistas sobre a qualidade das 20 lives mais visualizadas, sendo indicada a recomendação para utilização para fins acadêmicos e de aperfeiçoamento profissional. Foi estabelecido um ranking das dez lives mais recomendadas pelos especialistas. Toda a catalogação está disponibilizada neste trabalho ou na nuvem, podendo ser utilizada como base de consulta para a escolha de lives para diversas finalidades por docentes, discentes, profissionais e demais interessados.
\end{abstract}

Palavras-chave: Lives, Gestão e economia da construção, Ensino, Capacitação.

\section{ABSTRACT}

The Covid-19 pandemic changed the relationships between people, knowledge and teaching-learning. Then, lives/webinars that place during the pandemic, its relevance for the popularization of knowledge in Construction Management and Economics (CME) was investigated. The paper aims to catalog the lives included in this area and to make a qualitative analysis of the most viewed. Soon, a survey of lives was carried out on YouTube, categorizing them in the thematic lines of the CME and establishing a ranking of the most viewed. These main lives went through an evaluation process by experts in the field, using an Evaluation Form. As main results and contributions, 144 lives were cataloged, framed in the 14 thematic lines of the CME. Thus, 50 expert assessments were obtained about the quality of the 20 most viewed lives, indicating the recommendation for use for of academic and professional improvement. A ranking of the ten most recommended lives by experts was established. All cataloging can be used as a basis for consulting the choice of lives for various purposes by teachers, students, professionals and other interested.

Keywords: Lives, Construction management and economics, Teaching, Professional improvement.

${ }^{1}$ CRUZ, H. M.; AMARAL, T. G. do; SANTOS, D. N. dos; ALVES, M. R. S.; SANTOS, D. G.; HEINECK, L. F. M. Análise das lives enquadradas na área de gestão e economia da construção realizadas durante a pandemia da covid-19. In: SIMPÓSIO BRASILEIRO DE GESTÃO E ECONOMIA DA CONSTRUÇÃO, 12., 2021, Maceió. Anais[...] Porto Alegre: ANTAC, 2021. p.1-8. Disponível em: https://eventos.antac.org.br/index.php/sibragec/article/view/652. Acesso em: 2 out. 2021. 


\section{INTRODUÇÃO}

Trabalho remoto, educação à distância e comércio eletrônico intenso são transformações resultantes da pandemia nas relações de trabalho, no consumo e na educação. A velocidade com que os hábitos foram modificados diante da ameaça da pandemia provocada pelo coronavírus, trouxe transformações positivas que modificaram as tendências de consumo e de comportamento, que provavelmente aconteceriam de forma gradual e a longo prazo. Porém, nem mesmo os professores que já adotavam ambientes onlines como meios de ensino, imaginavam essa mudança tão rápida e de forma quase obrigatória, devido a pandemia (MOREIRA; HENRIQUE; BARROS, 2020).

No âmbito do ensino em arquitetura, engenharia e construção civil, as alternativas oferecidas incentivaram mudanças na didática até então empregada, assim como nas práticas profissionais.

De acordo com Freitas, Schimid e Tavarez (2014), as tecnologias de informação e comunicação contribuem de diferentes formas nas relações sociais, no entretenimento, na educação e no trabalho. No mundo do trabalho, atua de forma diferente com mais flexibilidade e liberdade, além de tornar a comunicação fluida e eficiente.

Diante desse cenário e necessidades, Santos e Nascimento (2021) destacam que o desenvolvimento tecnológico tem possibilitado a implementação de novos dispositivos no cotidiano das pessoas, proporcionando outros hábitos sociais. As mídias sociais são um dos elementos que se configuram nesse contexto, pois têm se tornado um fenômeno com características que também têm modificado a dinâmica social, na qual emergem novas práticas culturais, diferentes modos de interação e compartilhamento de informação em rede.

As mídias sociais são utilizadas por diversas instituições com finalidades variadas na produção de conteúdo. Calil, Corrêa e Spudeit (2013) argumentam que estas são ambientes que propiciam o compartilhamento de informações e podem obter com esses dispositivos, um forte aliado na disseminação de seus produtos e serviços, bem como aproveitar seu potencial de alcance para criar um canal de comunicação direta com seus interagentes que vai além de suas limitações de tempo e espaço.

Nesse contexto, partindo do pressuposto que essas novas formas de apresentação de conteúdo podem ser utilizadas para ensino de gestão e economia da construção, como um apoio às aulas de graduação e pós-graduação (stricto e lato sensu). Questiona-se se as lives mais visualizadas contribuem para o avanço na gestão da construção (GEC). Deste modo, o objetivo do presente artigo foi de catalogar as lives enquadradas na área de Gestão e Economia da Construção e realizar uma análise qualitativa daquelas mais visualizadas.

Decidiu-se por realizar a pesquisa a partir do levantamento das lives na plataforma de vídeos "Youtube", pelo fato da mesma ser de acesso livre (sem necessidade de registro/criação de conta) e também ser a maior plataforma de vídeos. O número de pessoas conectadas na internet é de aproximadamente 4,0 bilhões, sendo o YouTube a maior plataforma de vídeos com cerca de 2,0 bilhões de usuários (YOUTUBE, 2021).

\section{METODOLOGIA}

O estudo enquadra-se em um levantamento de campo e aplicação de questionário com especialistas, com apoio de pesquisa bibliométrica. É importante ressalvar que o levantamento de campo compreendeu as buscas nas mídias sociais de lives e ou webinários, aqui denominados todos de lives.

Para a busca, foram definidos os filtros a serem aplicados, de acordo com a ferramenta de busca do Youtube: duração longa e ordenada por relevância. Outro passo, foi a escolha 
das palavras-chaves que eram relacionadas aos títulos e principais temas abordados para cada uma das 14 linhas temáticas da área de Gestão e Economia da Construção (GEC) segundo a ANTAC $^{2}$. As palavras-chaves utilizadas estão disponíveis na planilha "Levantamento de Lives - GEC", mencionada em Resultados.

Iniciadas as buscas, os resultados passaram por uma segunda filtragem manual: enquadramento como lives ou webinars, relação com algumas das linhas temáticas da Gestão e Economia da Construção e data de ocorrência entre março de 2020 e março de 2021.

Os vídeos que passaram por estas duas filtragens foram catalogados em planilha eletrônica e foram colhidas as seguintes informações: título, participantes, canal do Youtube e quantidade de visualizações.

Após a catalogação foi possível realizar a divisão dos vídeos por linhas temáticas e o ranqueamento a partir da quantidade de visualizações; desta forma, foi elaborada uma lista com as 20 lives mais visualizadas, sendo composta com no mínimo uma representante de cada linha temática. Esta lista, então, foi disponibilizada para uma avaliação qualiquantitativa por especialistas (engenheiros civis ou arquitetos que atuam como docentes, pesquisadores ou profissionais de empresas de construção).

Os avaliadores foram convidados a preencher a Ficha de Avaliação ${ }^{3}$, escolhendo uma das lives para que assistissem e avaliassem e, ao final, indicassem livremente recomendações entre a lista geral das lives catalogadas.

A avaliação se deu por escala semântica, de 1 (totalmente insatisfeito) a 5 (totalmente satisfeito), para os seguintes itens: linguagem empregada; dinâmica da apresentação; didática dos participantes; conteúdo visual; duração; aprofundamento da abordagem do tema; conceitos técnicos empregados; exemplificações práticas; perfil profissional dos participantes; credibilidade da instituição promotora; experiência compartilhada. Ao fim, os respondentes indicaram o quanto recomendaria a live avaliada para fins acadêmicos (ser utilizada como material didático complementar de aulas, discussões de grupos acadêmicos etc.) e de aperfeiçoamento profissional (ser utilizada por empresas, órgãos ou por profissionais para seu aperfeiçoamento).

Para facilitar a discussão e visualização dos resultados, durante o tratamento dos dados, foram calculadas as médias para cada item de avaliação e as mesmas foram também convertidas em uma escala ilustrativa (estrelas), com o seguinte intervalo correspondente: 1 a $1,49=1$ estrela; 1,5 a 2,49 = 2 estrelas; 2,5 a 3,49 = 3 estrelas; 3,5 a 4,49 = 4 estrelas; 4,5 a $5=5$ estrelas.

\section{RESULTADOS}

\subsection{Caracterização geral do levantamento}

A planilha "Levantamento de Lives - GEC"4 apresenta o resultado da catalogação realizada no Youtube contendo 144 lives (ordenadas por número de visualizações) divididas nas 14 linhas temáticas da Gestão e Economia da Construção, com informações sobre o respectivo canal do Youtube, onde se encontra o vídeo, a instituição promotora, os participantes, o número de visualizações e o link de acesso às mesmas.

\footnotetext{
2 Dados da Associação Nacional de Tecnologia no Ambiente Construído. Disponível em: https://www.antac.org.br/gestaoecotemas

${ }^{3}$ Disponivel em: https://forms.gle/a2LgzUezuqQfV7AT6

4 Disponível em:

https://docs.google.com/spreadsheets/d/lgEEFVhluPk3Zh5LktASWEttDbzN_LLwGtoH31dDAzGs/e dit? usp=sharing
} 
O Quadro 1 traz uma caracterização geral do levantamento, contendo a quantidade de lives por linha temática e a instituição promotora.

Observa-se que a linha temática TIC's foi aquela com a maior quantidade de lives (21,5\%), seguida de Gestão da Produção (13,2\%) e Gestão da SST (12,5\%). Gestão de Riscos, Gestão da Cadeia de Suprimentos e Logística, além de Gestão do Conhecimento e da Informação, representaram juntas menos de 5\% das 144 lives catalogadas no Youtube.

Analisando a distribuição das origens das lives, pôde-se observar que instituições empresariais foram as que promoveram mais lives (41\%), seguidas de instituições diversas (33\%). A academia foi responsável pelo menor percentual de promoção (26\%), porém, é válido destacar que muitos docentes e discentes participaram como convidados/palestrantes de diversas lives promovidas por empresas.

Quadro 1 - Caracterização geral do levantamento

\begin{tabular}{|c|c|c|c|c|c|c|}
\hline \multirow{2}{*}{\multicolumn{2}{|c|}{ Linh a temática }} & \multirow[b]{2}{*}{ Qtd. lives } & \multirow[b]{2}{*}{$\%$ lives } & \multicolumn{3}{|c|}{ Instituição p romotora } \\
\hline & & & & \multirow{2}{*}{$\begin{array}{c}\text { Academia } \\
11\end{array}$} & \multirow{2}{*}{$\begin{array}{c}\text { Empress } \\
13\end{array}$} & \multirow{2}{*}{\begin{tabular}{|c|}
$\begin{array}{c}\text { Diversas } \\
\text { (Youtubers'a } \\
\text { utônomos) }\end{array}$ \\
7 \\
\end{tabular}} \\
\hline TIC's & $\begin{array}{c}\text { Tecnologias da Informação e } \\
\text { Comunicação aplicadas à } \\
\text { Gestão da Construção }\end{array}$ & 31 & $21,5 \%$ & & & \\
\hline Produção & Gestão da Produção & 19 & $13,2 \%$ & 3 & 9 & 7 \\
\hline G. SST. & $\begin{array}{c}\text { Gestão da Saúde e } \\
\text { Sequrança do Trabalho }\end{array}$ & 18 & $12,5 \%$ & 7 & 5 & 6 \\
\hline Custos & Gestão de Custos & 12 & $8,3 \%$ & 0 & 5 & 7 \\
\hline Estr. Organiz. & $\begin{array}{c}\text { Gestão Estratégica e } \\
\text { Organizacional }\end{array}$ & 11 & $7,6 \%$ & 2 & 5 & 4 \\
\hline Contratos & $\begin{array}{c}\text { Gestão de Contratos e de } \\
\text { Aquisicões }\end{array}$ & 9 & $6,3 \%$ & 2 & 5 & 2 \\
\hline Ensino & $\begin{array}{c}\text { Ensino de Gestão da } \\
\text { Construção }\end{array}$ & 9 & $6,3 \%$ & 6 & 1 & 2 \\
\hline Inov. & Gestão da Inovação & 9 & $6,3 \%$ & 1 & 6 & 2 \\
\hline Manut. e Oper. & $\begin{array}{c}\text { Gestão da Manutenção e de } \\
\text { Operacões }\end{array}$ & 7 & $4,9 \%$ & 1 & 3 & 3 \\
\hline Quali & Gestão da Qualidade & 6 & $4,2 \%$ & 1 & 2 & 3 \\
\hline $\begin{array}{l}\text { G. Sust. } \\
\text { Canteiros }\end{array}$ & $\begin{array}{c}\text { Gestão Sustentável nas } \\
\text { Empresas e Canteiros de } \\
\text { Obra }\end{array}$ & 6 & $4,2 \%$ & 2 & 2 & 2 \\
\hline G. Riscos & Gestão de Riscos & 3 & $2,1 \%$ & 1 & 0 & 2 \\
\hline Supri. e Log. & $\begin{array}{c}\text { Gestão da Cadeia de } \\
\text { Suprimentos e Logística }\end{array}$ & 3 & $2,1 \%$ & 1 & 2 & 0 \\
\hline Conheci. e Info. & $\begin{array}{l}\text { Gestão do Conhecimento e } \\
\text { da Informação }\end{array}$ & 1 & $0,7 \%$ & 0 & 1 & 0 \\
\hline & & 144 & & 38 & 59 & 47 \\
\hline & & & & $26 \%$ & $41 \%$ & $33 \%$ \\
\hline
\end{tabular}

Fonte: Os autores (2021)

\subsection{Avaliações qualitativas das lives}

A partir desse levantamento, foi realizado um ranking das 20 lives mais visualizadas que foram submetidas à avaliação de especialistas (Quadro 2). Ao todo, foram recebidas 50 avaliações.

É possível observar que as linhas temáticas TIC's e Gestão Estratégica e Organizacional foram as que tiveram mais lives, compondo este ranking, cada uma com três representações. A live mais visualizada foi relativa à Gestão da Qualidade (38.385 visualizações), seguida de uma live classificada na linha de Gestão de SST (14.290 visualizações) e de outra enquadrada na linha de TIC`s (5.406 visualizações). 
Quadro 2 - Ranking das 20 lives mais visualizadas e respectivas avaliações qualitativas

\begin{tabular}{|c|c|c|c|c|c|c|c|c|}
\hline \multirow[b]{2}{*}{ Ordem } & \multirow[b]{2}{*}{ Titulo } & \multirow[b]{2}{*}{$\begin{array}{l}\text { Canal/Inst. } \\
\text { Promotora }\end{array}$} & \multirow[b]{2}{*}{ Palestrantes } & \multirow[b]{2}{*}{ Linha temática GEC } & \multirow[b]{2}{*}{$\begin{array}{c}\text { Visualiza- } \\
\text { ções }\end{array}$} & \multirow{2}{*}{$\begin{array}{c}\text { Avaliação } \\
\text { geral }\end{array}$} & \multicolumn{2}{|c|}{ Indicaçäo de finalidade } \\
\hline & & & & & & & Acadêmica & $\begin{array}{c}\text { Aperf. } \\
\text { profissional }\end{array}$ \\
\hline 1 & $\begin{array}{c}\text { Sistema americano de } \\
\text { gestão da qualidade na } \\
\text { construção civil para } \\
\text { construtoras }\end{array}$ & $\begin{array}{l}\text { Construção } \\
\text { Civil }\end{array}$ & $\begin{array}{l}\text { Prof. Dr. Eng. Abdala Carim Nabut } \\
\text { Neto; Prof. Dr. Bernardo Tutikian }\end{array}$ & Gestão da Qualidade & 38385 & $\star^{\star} \star$ & $\star \star \star$ & $\star^{\star} \star$ \\
\hline 2 & $\begin{array}{c}\text { Programa de } \\
\text { Gerenciamento de Riscos - } \\
\text { PGR } \\
\end{array}$ & $\begin{array}{c}\text { FUNDACENT } \\
\text { RO }\end{array}$ & $\begin{array}{l}\text { Gilmar Trivelato (Pesquisador da } \\
\text { Fundacentro) }\end{array}$ & Gestão da SST & 14290 & $\star^{\star \star \star} \star$ & $\star^{\star} \star$ & $\star^{\star} \star \star \star$ \\
\hline 3 & $\begin{array}{l}\text { Gerenciando projetos } \\
\text { através do BIM }\end{array}$ & TV ABRAPLAN & $\begin{array}{c}\text { Gabriel Gonçalvez - Manager na } \\
\text { Deloitte }\end{array}$ & $\begin{array}{c}\text { TIC's aplicadas à } \\
\text { Gestão da Construção }\end{array}$ & 5406 & $\star \star$ & $\star \star$ & $\star^{\star} \star$ \\
\hline 4 & $\begin{array}{c}\text { Como gerenciar seu chefe } \\
\text { e criar um departamento de } \\
\text { inovação BM }\end{array}$ & $\begin{array}{c}\text { BIMExperts } \\
\text { Treinamentos }\end{array}$ & Artur Bessoni e Diego Bieger & Gestão da hovação & 5363 & $\star^{\star} \star \star$ & $\star^{\star} \star \star$ & $\star^{\star} \star$ \\
\hline 5 & $\begin{array}{c}\text { Os segredos do } \\
\text { gerenciamento de obras - } \\
\text { Com o Eng. Arq. Fabrício } \\
\text { Rossi } \\
\end{array}$ & $\begin{array}{l}\text { O Canal da } \\
\text { Engenharia }\end{array}$ & $\begin{array}{l}\text { Eng. Felipe Rodrigues e Eng. } \\
\text { Fabricio Rossi }\end{array}$ & Gestão da Produção & 3562 & $\star^{\star \star} \star$ & $\star^{\star}$ & $\star^{\star} \star$ \\
\hline 6 & $\begin{array}{c}\text { Webinar: ABNT NBR } 16747 \\
\text { Inspeção Predial: O que } \\
\text { muda na prática da } \\
\text { Inspeção Predial }\end{array}$ & IBAPE SP & $\begin{array}{l}\text { Antônio Carlos Dolacio; Sérgio } \\
\text { Levin; Fláva Andreatta Pujadas; } \\
\text { Alexandre Oliveira; Patricia } \\
\text { Bittencourt }\end{array}$ & $\begin{array}{c}\text { Gestão da Manutenção } \\
\text { e de Operações }\end{array}$ & 3536 & $\star^{\star \star \star} \star$ & & $\star^{\star \star \star} \star$ \\
\hline 7 & $\begin{array}{c}\text { Como funciona a estratégia } \\
\text { das grandes construtoras } \\
\text { para Financiamentos } \\
\text { Imobiliários. }\end{array}$ & \begin{tabular}{|l|} 
Luciano Dual - \\
Financie Certo \\
\end{tabular} & Luciano Dual & $\begin{array}{c}\text { Gestão Estratégica e } \\
\text { Organizacional }\end{array}$ & 2762 & $\star \star$ & $\star$ & $\star \star$ \\
\hline 8 & $\begin{array}{c}\text { Canteiros Inteligentes: } \\
\text { Tecnologias digitais } \\
\text { aplicadas aos canteiros de } \\
\text { obras } \\
\end{array}$ & Enredes & $\begin{array}{c}\text { Gustavo Aguiar (Método), Renato } \\
\text { Genioli (R. Yazbek), Franco Elmor } \\
\text { (MRV), Ana Csestak (Autodoc) e } \\
\text { Roberto Souza (Enredes) } \\
\end{array}$ & $\begin{array}{c}\text { TIC's aplicadas à } \\
\text { Gestão da Construção }\end{array}$ & 2620 & $\star^{\star} \star$ & $\star^{\star} \star$ & $\star^{\star} \star \star$ \\
\hline 9 & $\begin{array}{c}\text { Construção Civil em } \\
\text { Tempos de Coronavírus (10 } \\
\text { Webinar) }\end{array}$ & $\begin{array}{l}\text { SEBRAE } \\
\text { MINAS }\end{array}$ & & $\begin{array}{c}\text { Gestão Estratégica e } \\
\text { Organizacional }\end{array}$ & 2177 & $\star \star$ & $\star \star$ & $\star \star$ \\
\hline 10 & $\begin{array}{c}\text { Webinar: Last Planner -A } \\
\text { Essência do Planejamento } \\
\text { e Controle de Obras }\end{array}$ & $\begin{array}{l}\text { Produttare } \\
\text { Consultoria }\end{array}$ & $\begin{array}{l}\text { Professor Carlos Torres Formoso - } \\
\text { (UFRGS), Eduardo de Oliveira } \\
\text { (Produttare); Junico Antunes } \\
\text { (Produttare) }\end{array}$ & Gestão da Produção & 1926 & & & $\star^{\star^{*}} \star^{\star} \star$ \\
\hline 11 & $\begin{array}{c}\text { Como o ensino a distância } \\
\text { pode afetar na formação do } \\
\text { engenheiro civil? }\end{array}$ & $\begin{array}{c}\text { Bloco } \\
\text { Construções }\end{array}$ & \begin{tabular}{|c|} 
Eng $^{0}$ Civil Paulo Sérgio Carvalho \\
(PS Videoaulas) ; Riccelly Lacerda \\
(Bloco Construções)
\end{tabular} & $\begin{array}{l}\text { Ensino de Gestão da } \\
\text { Construção }\end{array}$ & 1905 & $\star^{\star} \star$ & $\star^{\star} \star$ & $\star \star$ \\
\hline 12 & $\begin{array}{l}\text { CGE ORIENTA - } 4^{\circ} \text { CICLO - } \\
\text { Gestão e Fiscalização de } \\
\text { Contratos e Obras }\end{array}$ & \begin{tabular}{|c|} 
Controladoria \\
Geral do \\
Estado de \\
Mato Grosso \\
\end{tabular} & & $\begin{array}{c}\text { Gestão de Contratos e } \\
\text { de Aquisições }\end{array}$ & 1863 & $\star^{\star} \star \star$ & $\star$ & $\star$ \\
\hline 13 & $\begin{array}{c}\text { BIM: Desafios Reais da } \\
\text { Adoção e Implantação da } \\
\text { Metodologia } \\
\end{array}$ & SIENGE & $\begin{array}{c}\text { Jeferson Böes; Julio Carneiro; } \\
\text { Leonardo Dias de Santana; Giseli } \\
\text { Barbosa Anversa } \\
\end{array}$ & $\begin{array}{c}\text { TIC's aplicadas à } \\
\text { Gestão da Construção }\end{array}$ & 1710 & $\star \star$ & $\star \star \star$ & $\star$ \\
\hline 14 & $\begin{array}{l}\text { Afinal, o que é o } \\
\text { Aprovisionamento? }\end{array}$ & Herbert Viana & Prof. Hebert Viana & $\begin{array}{c}\text { Gestão da Manutenção } \\
\text { e de Operações }\end{array}$ & 1587 & $\star^{\star} \star$ & $\star^{\star} \star \star$ & $\star^{\star} \star$ \\
\hline 15 & $\begin{array}{c}\text { Filosofia Lean } \\
\text { impulsionando a gestão das } \\
\text { empresas da construção } \\
\text { civil }\end{array}$ & Enredes & 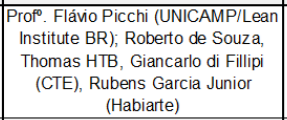 & $\begin{array}{l}\text { Gestão Estratégica e } \\
\text { Organizacional }\end{array}$ & 1567 & & & \\
\hline 16 & $\begin{array}{l}\text { Montagem de um } \\
\text { orçamento no Sienge }\end{array}$ & $\begin{array}{c}\text { Bloco } \\
\text { Construções }\end{array}$ & $\begin{array}{l}\text { Riccelly Lacerda (Bloco } \\
\text { Construções) } \\
\end{array}$ & Gestão de Custos & 1109 & $\star \star$ & $\star$ & $\star \star \star$ \\
\hline 17 & $\begin{array}{l}\text { Gestão Sustentável de } \\
\text { Obras }\end{array}$ & $\begin{array}{l}\text { Eliane - } \\
\text { CiGOUFU }\end{array}$ & $\begin{array}{c}\text { Profa. Dayana Bastos Costa } \\
\text { (UFBA); Eng Gustavo Adriano } \\
\text { Coura (Alphaville Urbanismo); } \\
\text { Profa. Eliane Betanea e Luiza } \\
\text { Yamada (FEVIC/UFU) }\end{array}$ & $\begin{array}{l}\text { Gestão Sustentável } \\
\text { nas Empresas e } \\
\text { Canteiros de Obra }\end{array}$ & 568 & & & $\star^{\star \star}$ \\
\hline 18 & $\begin{array}{l}\text { Webinar: Last Planner -A } \\
\text { Essência do Planejamento } \\
\text { e Controle de Obras }\end{array}$ & $\begin{array}{l}\text { Produttare } \\
\text { Consultoria }\end{array}$ & $\begin{array}{c}\text { Profo Carlos Torres Formoso - } \\
\text { (UFRGS), Eduardo de Oliveira } \\
\text { (Produttare); Junico Antunes } \\
\text { (Produttare) }\end{array}$ & $\begin{array}{l}\text { Gestão da Cadeia de } \\
\text { Suprimentos e } \\
\text { Logística }\end{array}$ & 561 & & $\star^{\star \star \star} \star$ & $\star^{\star \star \star} \star$ \\
\hline 19 & $\begin{array}{c}\text { Gestão visual na } \\
\text { construção civil: conceitos e } \\
\text { boas práticas - Webinar }\end{array}$ & $\begin{array}{l}\text { Produttare } \\
\text { Consultoria }\end{array}$ & \begin{tabular}{|c|} 
Caroline Porto Valente (UTS- \\
Sidney), Profo Carlos Torres \\
Formoso (NORIE-UFRGS), Eduardo \\
de Oliveira (Produttare); Junico \\
Antunes(Produttare) \\
\end{tabular} & $\begin{array}{l}\text { Gestão do } \\
\text { Conhecimento e da } \\
\text { Informação }\end{array}$ & 311 & $\star^{*}{ }^{\star}$ & & $x^{k^{\star}} \star \star x$ \\
\hline 20 & $\begin{array}{l}\text { Gestão de riscos: teoria e } \\
\text { prática na construção civil }\end{array}$ & $\begin{array}{c}\text { Carreira } \\
\text { Engenharia } \\
\text { Civil } \\
\end{array}$ & Eng. Prof. André Abreu Paim & Gestão de Riscos & 242 & $\star^{\star \star} \star$ & $\star^{\star} \star$ & $\star^{\star \star} \star$ \\
\hline & 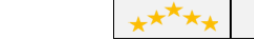 & 3 lives & $\star^{\star} \star \star$ & $\star \star$ & $\star$ & nenhuma & & \\
\hline
\end{tabular}

Fonte: Os autores (2021)

Quanto às avaliações pelos especialistas, a maior parte das lives foram bem avaliadas, como pode ser observado na parte inferior do Quadro 2. Nove lives receberam avaliações acima de quatro estrelas, já quatro lives receberam duas estrelas.

Ao buscar uma relação entre a quantidade de visualizações e a avaliação qualitativa das lives, foi plotado o gráfico explicitado na Figura 1. A análise gráfica sugere que o número de visualizações das lives não apresentou relação com a avaliação qualitativa das mesmas realizadas pelos especialistas. 
Figura 1 - Quantidade de visualizações $(\log )$ versus média das avaliações

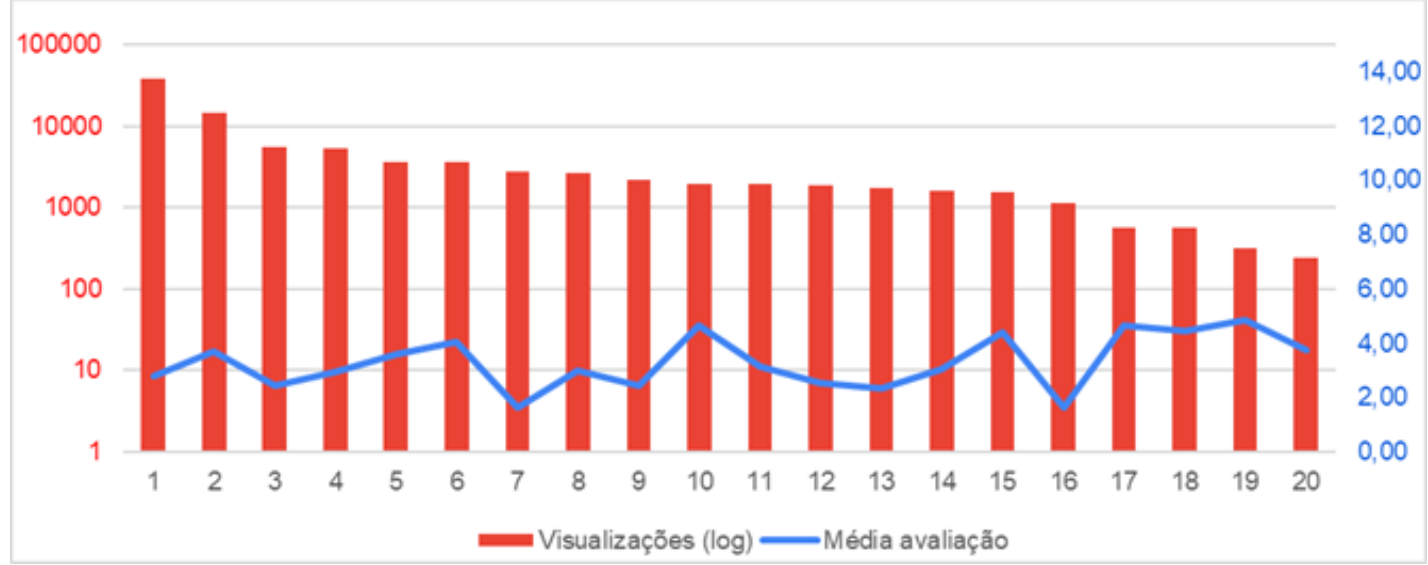

Fonte: Os autores (2021)

A observação do Quadro 2, por exemplo, mostra que as lives que receberam melhores avaliações (cinco estrelas) estão na $10^{\mathrm{a}}, 17^{\mathrm{a}}$ e $19^{\mathrm{a}}$ posições do ranking de visualizações. Outro aspecto a ser destacado é que estas três lives supracitadas tiveram como participantes profissionais da academia.

Os especialistas também foram convidados a responder o quanto indicavam a live, objeto de avaliação, para uso em fins acadêmicos e de aperfeiçoamento profissional. Percebe-se que as lives mais bem avaliadas tiveram também boas indicações para ambas as finalidades e que, de maneira geral, aquelas lives que receberam boas indicações para uma finalidade também receberam para a outra.

Durante o processo de avaliação pelos especialistas, os mesmos foram convidados a fazer recomendações livremente dentre as 144 lives catalogadas. Ao todo, foram recomendadas 40 lives. Adiante, o Quadro 3 traz as dez lives mais recomendadas pelos especialistas. 
Quadro 3 - As dez lives mais recomendadas pelos especialistas ${ }^{5}$

\begin{tabular}{|c|c|c|c|c|c|}
\hline $\begin{array}{l}\text { Código } \\
\text { da live }\end{array}$ & Título da Live & Autor/Canal & Participantes & Linha temática & $\begin{array}{c}\text { № } \\
\text { recomen- } \\
\text { dações }\end{array}$ \\
\hline 13 & $\begin{array}{l}\text { Webinar: Last Planner - A Essência do } \\
\text { Planejamento e Controle de Obras }\end{array}$ & $\begin{array}{l}\text { Produttare } \\
\text { Consultoria }\end{array}$ & $\begin{array}{c}\text { Carlos Formoso - (UFRGS), Eduardo } \\
\text { de Oliveira (Produttare); Junico Antunes } \\
\text { (Produttare) }\end{array}$ & $\begin{array}{l}\text { Gestão da } \\
\text { Produção }\end{array}$ & 6 \\
\hline 14 & Construção Enxuta na Prática & $\begin{array}{l}\text { GT Gestão e } \\
\text { Economia da } \\
\text { Construção }\end{array}$ & $\begin{array}{c}\text { Clarissa Biotto (UFSCar), Bruno P. } \\
\text { Mota (Logikal Projects) }\end{array}$ & $\begin{array}{l}\text { Gestão da } \\
\text { Produção }\end{array}$ & 5 \\
\hline 7 & $\begin{array}{l}\text { Melhores práticas e necessidades de } \\
\text { avanços em gestão sob a ótica de } \\
\text { profissionais atuantes no mercado de } \\
\text { construção }\end{array}$ & $\begin{array}{l}\text { GT Gestão e } \\
\text { Economia da } \\
\text { Construção }\end{array}$ & $\begin{array}{c}\text { Maria Angelica Covelo Silva (NGI } \\
\text { Consultoria), Alexandre Britez (GP\&D } \\
\text { Consultoria e Projetos), Rogerio Sato } \\
\text { (Assahi \& Associados), Ana Cristina } \\
\text { Catai Chalita (HTB Engenharia e } \\
\text { Construção), Ubiraci Espinelli Lemes } \\
\text { de Souza (POLI-USP), Ricardo Oviedo } \\
\text { Haito (UFSC), Sávio Melo (UFBA) }\end{array}$ & $\begin{array}{c}\text { Gestão Estratégica } \\
\text { e Organizacional }\end{array}$ & 3 \\
\hline 17 & $\begin{array}{l}\text { [Webinar]Gestão da produção com } \\
\text { Lean Construction: as alavancas para a } \\
\text { produtividade no seu canteiro }\end{array}$ & Aval & $\begin{array}{l}\text { Eng. Aldo Dorea Mattos, Eng. André } \\
\text { Quinderé (Aval Engenharia) }\end{array}$ & $\begin{array}{l}\text { Gestão da } \\
\text { Produção }\end{array}$ & 3 \\
\hline 19 & $\begin{array}{l}\text { Planejamento e Controle Baseado em } \\
\text { Localização na Construção Civil }\end{array}$ & $\begin{array}{l}\text { INOVACON } \\
\text { CEARÁ }\end{array}$ & Carlos Formoso (UFGRS) & $\begin{array}{l}\text { Gestão da } \\
\text { Produção }\end{array}$ & 3 \\
\hline 84 & $\begin{array}{l}\text { GETEC LIVE 04.06.2020: Como } \\
\text { integrar BIM e a Internet das Coisas na } \\
\text { indústria da Construção Civil? }\end{array}$ & GETEC-UFBA & $\begin{array}{l}\text { Emerson de Andrade M. Ferreira } \\
\text { (UFBA); Sávio Melo (UFBA) }\end{array}$ & $\begin{array}{c}\text { Tecnologias da } \\
\text { Informação e } \\
\text { Comunicação } \\
\text { aplicadas à Gestão } \\
\text { da Construção }\end{array}$ & 3 \\
\hline 3 & $\begin{array}{c}\text { Webinar } 18 \text { | Filosofia Lean } \\
\text { impulsionando a gestão das empresas } \\
\text { da construção civil }\end{array}$ & Enredes & $\begin{array}{l}\text { Roberto de Souza Thomas (HTB), } \\
\text { Giancarlo di Fillipi (CTE), Rubens } \\
\text { Garcia Junior (Habiarte), Flávio Picchi } \\
\text { (UniCamp/Lean Institute) }\end{array}$ & $\begin{array}{c}\text { Gestão Estratégica } \\
\text { e Organizacional }\end{array}$ & 2 \\
\hline 6 & $\begin{array}{l}\text { A produtividade como estratégia de } \\
\text { negócio }\end{array}$ & INOVACON CE & $\begin{array}{l}\text { Luiz Henrique Ceotto (USP/Urbic); } \\
\text { Barros Neto (UFC); Roberto Dudma } \\
\text { (Inovacon) ; Jorge Dantas (Inovacon) }\end{array}$ & $\begin{array}{l}\text { Gestão Estratégica } \\
\text { e Organizacional }\end{array}$ & 2 \\
\hline 8 & $\begin{array}{l}\text { A Jornada Lean rumo ao Sistema de } \\
\text { Produção da Camargo Corrêa Infra }\end{array}$ & $\begin{array}{l}\text { Produttare } \\
\text { Consultoria }\end{array}$ & $\begin{array}{l}\text { Renato Furtado (Camargo \& Correia) } \\
\text { Junico Antunes (Produttare) ; Eduardo } \\
\text { de Oliveira (Produttare) }\end{array}$ & $\begin{array}{c}\text { Gestão Estratégica } \\
\text { e Organizacional }\end{array}$ & 2 \\
\hline 15 & Construção Enxuta & $\begin{array}{l}\text { Eliane - } \\
\text { CiGOUFU }\end{array}$ & $\begin{array}{l}\text { Profa. Eliane Betênea (UFU), Clarissa } \\
\text { Biotto (UFSCar) e Eng. Fernando } \\
\text { Mendes (Alphaville Urbanismo) }\end{array}$ & $\begin{array}{l}\text { Gestão da } \\
\text { Produção }\end{array}$ & 2 \\
\hline
\end{tabular}

Fonte: Os autores (2021)

\section{CONSIDERAÇÕES FINAIS}

Foram levantadas 144 lives de interesse para a área de gestão e economia da construção, que podem ser visualizadas pelos interessados (catálogo compartilhado nesse artigo e na nuvem a partir dos links). Estas listas podem ser usadas para o ensino e ou capacitação, corroborando com a difusão de conhecimento em gestão da construção.

Para responder à questão do quão essas lives contribuem para o avanço na gestão da construção (GEC), e com isso apontar as que trazem mais contribuições, foram destacadas as 20 mais visualizadas para a avaliação dos especialistas. Verificou-se que o número de visualizações não é necessariamente o indicador mais adequado a ser considerado ao selecionar lives para serem utilizadas para fins de ensino ou de aperfeiçoamento profissional. Isto porque este número pode estar ligado a diversos fatores: quantidade de inscritos no canal promotor do Youtube, forma de divulgação e até o tipo de público que a live se propôs a alcançar. A partir dos resultados das avaliações as mais visualizadas diferem das que mais contribuem, na percepção de especialistas, para a GEC.

Ademais, com a pesquisa realizada constatou-se que as lives produzidas durante o período de pandemia (março de 2020 a março de 2021) foram promovidas, em sua maioria, por empresas, porém, mesmo essas, tiveram a participação de docentes e pesquisadores na função de mediadores e ou palestrantes.

\footnotetext{
${ }^{5}$ Tabela completa com todas as lives recomendadas e com os links acessíveis disponível em: https://docs.google.com/spreadsheets/d/1gEEFVhluPk3Zh5LktASWEttDbzN_LLwGtoH31dDAzGs/edit?usp=sharin 
Em relação aos limites da pesquisa, ressalta-se que foi utilizada somente a base do Youtube, desta forma, é importante frisar lives relevantes que ocorreram em outras mídias sociais (Instagram, Facebook dentre outras) podem não ter sido catalogadas. Neste âmbito, cabe como sugestão de trabalhos futuros a criação de um mecanismo permanente de catalogação e avaliação de lives, abrangendo inclusive estas outras mídias sociais.

\section{REFERÊNCIAS}

CALIL JUNIOR, A.; CORRÊA, E. C. D.; SPUDEIT, D. O uso das mídias sociais nas bibliotecas brasileiras: análise dos trabalhos apresentados no SNBU e CBBD. In: CONGRESSO BRASILEIRO DE BIBLIOTECONOMIA, DOCUMENTAÇÃO E CIÊNCIA DA INFORMAÇÃO, Florianópolis, 2013. Anais [...] Florianópolis, 2013, p.5044-5059.

FREITAS, M.C.D.; SCHMID, A. L.; TAVARES, S. F. Estratégia na comunicação científica na forma de vídeo pôster. In: Administração, direito e tecnologia: a serviço da cidadania. Curitiba: Instituto Memoria, 2014, p. 220-246.

SANTOS, A. P.; NASCIMENTO, V. G. Ação cultural com mídias sociais: análise do Facebook do Sistema Estadual de Bibliotecas Públicas de São Paulo (SisEB). ATOZ: NOVAS PRÁTICAS EM INFORMAÇÃO E CONHECIMENTO, v. 10, n. 1 (2021). jan./abr. São Paulo, 2021.

MOREIRA, J. A.; HENRIQUES, S.; BARROS, D. Transitando de um ensino remoto emergencial para uma educação digital em rede, em tempos de pandemia. Dialogia, 34, p.351-364. 2020.

YOUTUBE. Imprensa. Abril 2021. Disponível em: <https: //www.youtube.com/intl/ptBR/yt/about/press/>. Acesso em: 20 de abril 2021. 